\title{
Room Temperature Ionic Liquids from 20 Natural Amino Acids
}

\author{
Kenta Fukumoto, Masahiro Yoshizawa, and Hiroyuki Ohno
}

1-Ethyl-3-methylimidazolium L-a-aminopropionic acid salt ([emim][Ala]). From 1.0g (5.2mmol) of [emim] $[\mathrm{Br}]$ and $0.6 \mathrm{~g}(6.7 \mathrm{mmol})$ of L-alanine, $0.9 \mathrm{~g}$ of [emim][Ala] was obtained (Yield : 86\%). ${ }^{1} \mathrm{H}-\mathrm{NMR}$ (DMSO, $\delta / p p m$ relative to TMS) : 0.96(d, 3H, J=3.5 Hz), 1.35(t, 3H, J=7Hz), 2.80(q, 1H, J=10.3Hz), 3.83(s, 3H), 4.17(q, 2H, J=11.3Hz), 7.72(s, 1H), 7.81(s, 1H), 9.83(s, 1H). Elemental analysis. Calcd for $\mathrm{C}_{9} \mathrm{H}_{17} \mathrm{~N}_{3} \mathrm{O}_{2} 2 \mathrm{H}_{2} \mathrm{O}: \mathrm{C}, 45.93 ; \mathrm{H}, 9.01 ; \mathrm{N}, 17.86 ; \mathrm{O}, 27.20$. Found: C,45.92 ; H,8.74 ; N, 17.40 .0

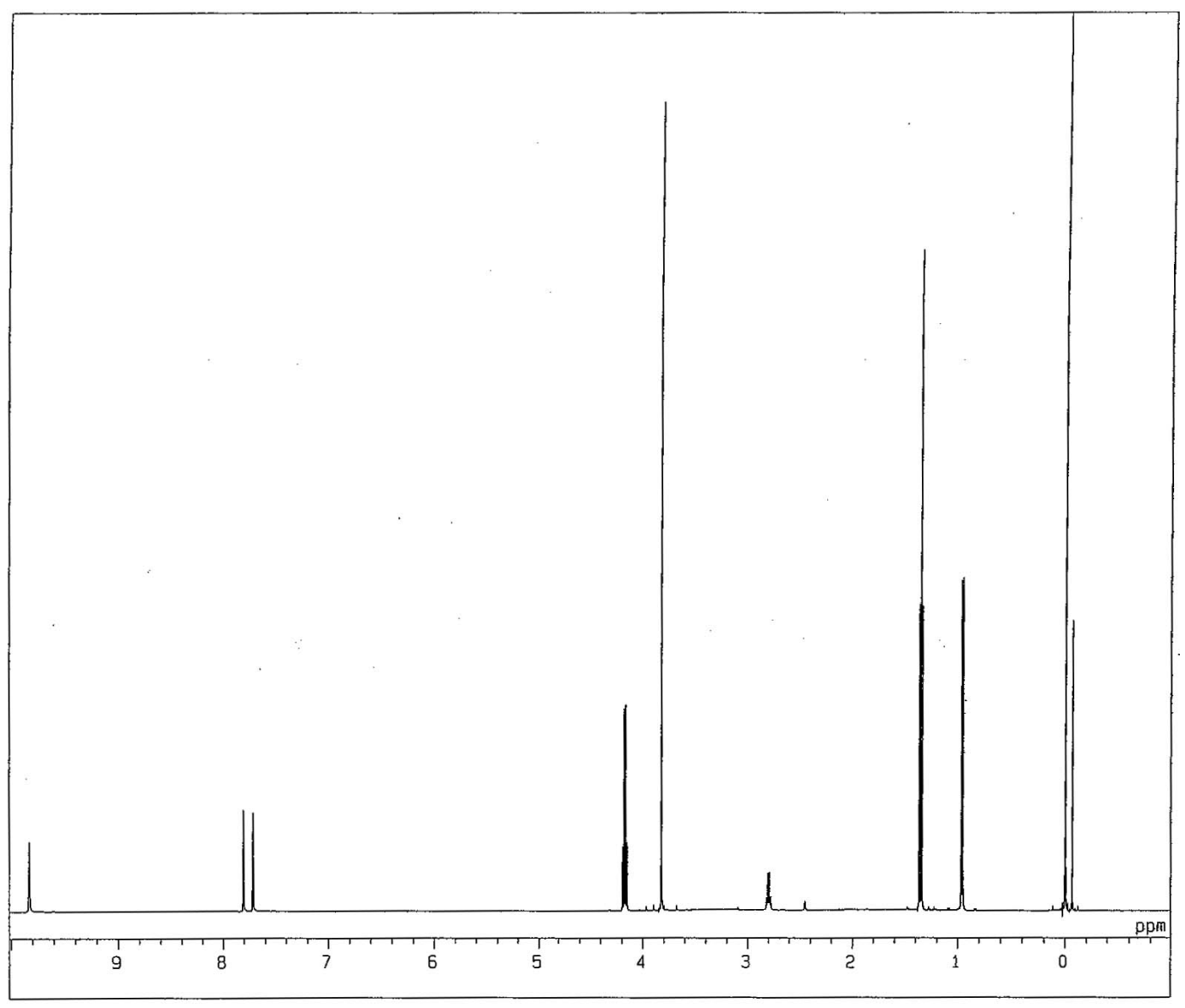


1-Ethyl-3-methylimidazolium 2-amino-5-guanidinovaleric acid salt ([emim][Arg]). From 1.5g

$(7.9 \mathrm{mmol})$ of $[\mathrm{emim}][\mathrm{Br}]$ and $1.6 \mathrm{~g}(9.2 \mathrm{mmol})$ of $\mathrm{L}$-arginine, $1.6 \mathrm{~g}$ of $[\mathrm{emim}][\mathrm{Arg}]$ was obtained (Yield :

74\%). ${ }^{1} \mathrm{H}-\mathrm{NMR}$ (DMSO, $\delta / \mathrm{ppm}$ relative to TMS) : $1.23(\mathrm{~m}, 2 \mathrm{H}, \mathrm{J}=15 \mathrm{~Hz}), 1.35(\mathrm{t}, 3 \mathrm{H}, \mathrm{J}=7 \mathrm{~Hz}), 1.44(\mathrm{~m}$,

$2 \mathrm{H}), 2.79(\mathrm{t}, 1 \mathrm{H}, \mathrm{J}=6.5 \mathrm{~Hz}), 2.85(\mathrm{t}, 2 \mathrm{H}, \mathrm{J}=9 \mathrm{~Hz}), 3.81(\mathrm{~s}, 3 \mathrm{H}), 4.16(\mathrm{q}, 2 \mathrm{H}, \mathrm{J}=11 \mathrm{~Hz}), 7.68(\mathrm{~d}, 1 \mathrm{H}, \mathrm{J}=1 \mathrm{~Hz})$,

7.77(d, $1 \mathrm{H}, \mathrm{J}=0.75 \mathrm{~Hz})$. Elemental analysis. Calcd for $\mathrm{C}_{12} \mathrm{H}_{24} \mathrm{~N}_{6} \mathrm{O}_{2} 1.5 \mathrm{H}_{2} \mathrm{O}: \mathrm{C}, 46.27 ; \mathrm{H}, 8.76 ; \mathrm{N}, 26.99$;

O, 17.98. Found: C, 46.32; H,8.68; N, 26.61.

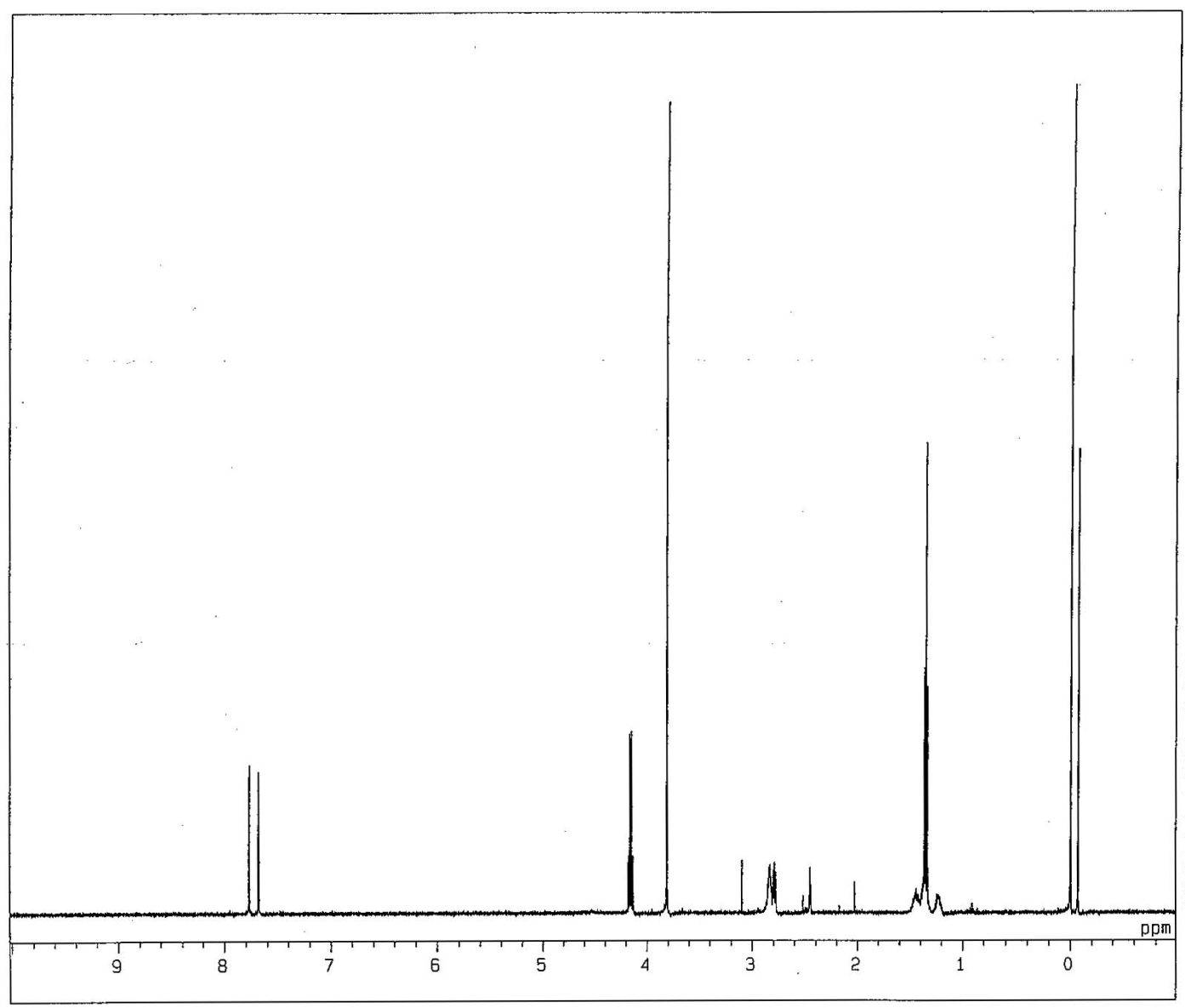


1-Ethyl-3-methylimidazolium $\boldsymbol{\alpha}$-aminosuccinamic acid salt ([emim][Asn]). From 1.0g (5.2mmol) of [emim] $[\mathrm{Br}]$ and $0.8 \mathrm{~g}(6.1 \mathrm{mmol})$ of L-asparagine, $1.1 \mathrm{~g}$ of [emim][Asn] was obtained (Yield : 83\%). ${ }^{1} \mathrm{H}-$ NMR (DMSO, $\delta / p p m$ relative to TMS) : 1.36(t, 3H, J=7.5Hz), 1.93(q, 1H, J=11.8Hz), 2.35(q, 1H, $\mathrm{J}=9.5 \mathrm{~Hz}), 3.06(\mathrm{q}, 1 \mathrm{H}, \mathrm{J}=6.3 \mathrm{~Hz}), 3.82(\mathrm{~s}, 3 \mathrm{H}), 4.16(\mathrm{q}, 2 \mathrm{H}, \mathrm{J}=10.8 \mathrm{~Hz}), 6.58(\mathrm{~s}, 1 \mathrm{H}), 7.70(\mathrm{t}, 1 \mathrm{H}, \mathrm{J}=2 \mathrm{~Hz})$, 7.78(t, $1 \mathrm{H}, \mathrm{J}=1.5 \mathrm{~Hz}), 7.99(\mathrm{~s}, 1 \mathrm{H}), 9.59(\mathrm{~s}, 1 \mathrm{H})$. Elemental analysis. Calcd for $\mathrm{C}_{10} \mathrm{H}_{18} \mathrm{~N}_{4} \mathrm{O}_{3} 0.9 \mathrm{H}_{2} \mathrm{O}: \mathrm{C}$, 46.45 ; H, 7.74; N, 21.68; O, 24.14. Found: C,46.67 ; H,7.75 ; N, 21.46.

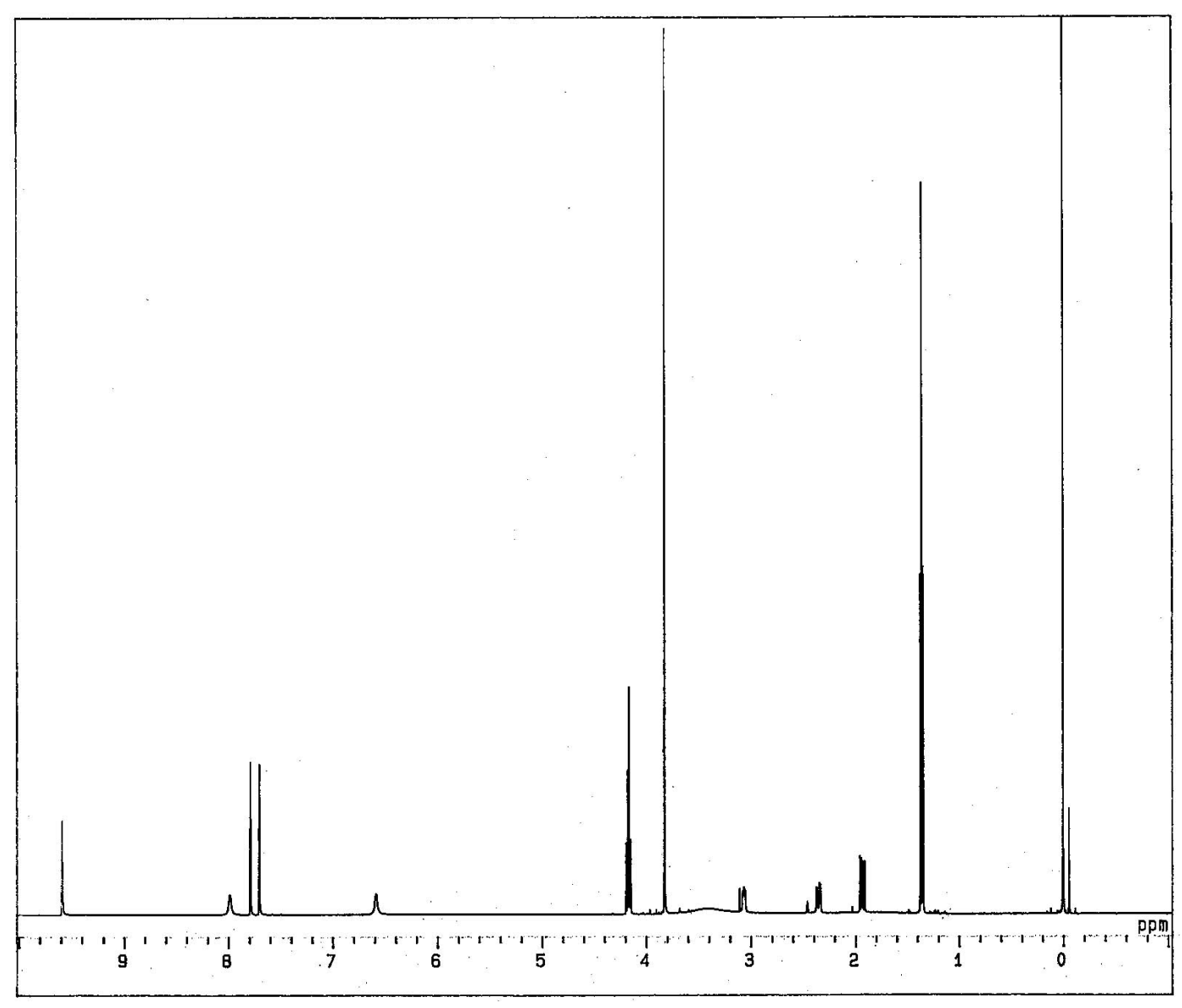


1-Ethyl-3-methylimidazolium aminosuccinic acid salt ([emim][Asp]). From $1.0 \mathrm{~g}$ (5.2mmol) of [emim] $[\mathrm{Br}]$, and $0.9 \mathrm{~g}(6.8 \mathrm{mmol})$ of L-asparatic acid, 1.1g of [emim][Asp] was obtained (Yield : 89\%). ${ }^{1} \mathrm{H}-\mathrm{NMR}$ (DMSO, $\delta / \mathrm{ppm}$ relative to TMS) : 1.35(t, 3H, J=7.5Hz), $2.03(\mathrm{~d}, 1 \mathrm{H}, \mathrm{J}=11.8 \mathrm{~Hz}), 2.25(\mathrm{~d}, 1 \mathrm{H}$, $\mathrm{J}=8.8 \mathrm{~Hz}), 3.16(\mathrm{t}, 1 \mathrm{H}, \mathrm{J}=7 \mathrm{~Hz}), 3.80(\mathrm{~s}, 3 \mathrm{H}), 4.14(\mathrm{q}, 2 \mathrm{H}, \mathrm{J}=10.8), 7.66(\mathrm{~s}, 1 \mathrm{H}), 7.74(\mathrm{~s}, 1 \mathrm{H}), 9.23(\mathrm{~s}, 1 \mathrm{H})$.

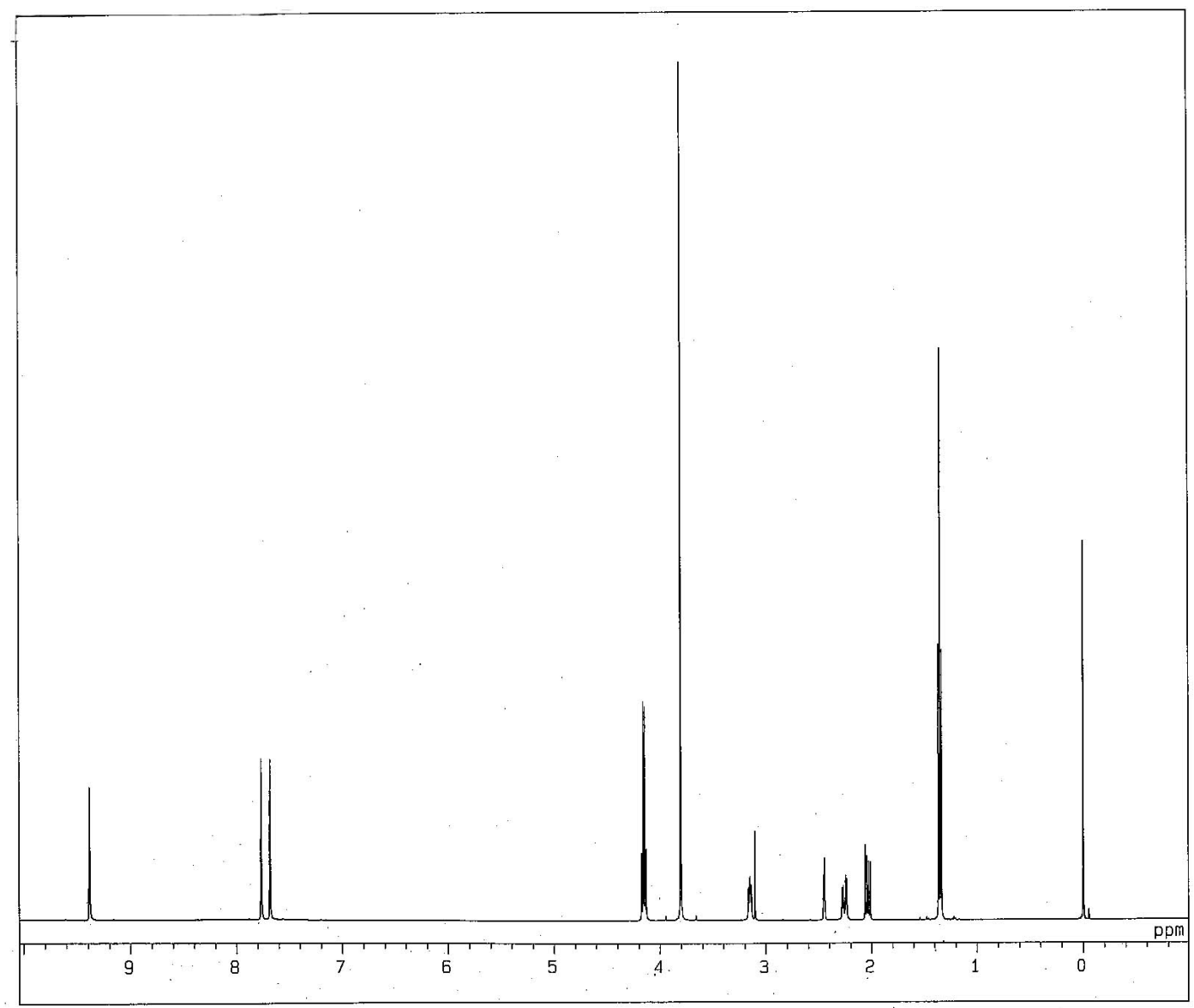


1-Ethyl-3-methylimidazolium 2-amino-3-mercaptopropionic acid salt ([emim][Cys]). The same procedure was used under nitrogen atmosphere. From $1.0 \mathrm{~g}(5.2 \mathrm{mmol})$ of $[\mathrm{emim}][\mathrm{Br}]$, and $0.8 \mathrm{~g}$ (6.6mmol) of L-cystein, $0.9 \mathrm{~g}$ of [emim] [Cys] was obtained (Yield : 77\%). ${ }^{1} \mathrm{H}-\mathrm{NMR}$ (DMSO, $\delta / \mathrm{ppm}$ relative to TMS ) : 1.36(t, 3H, J=7.5Hz), 2.45(d, 1H, J=10.3Hz), 2.67(d, $1 \mathrm{H}, \mathrm{J}=8.5 \mathrm{~Hz}), 2.93(\mathrm{t}, 1 \mathrm{H}$, $\mathrm{J}=5.5 \mathrm{~Hz}), 3.82(\mathrm{~s}, 3 \mathrm{H}), 4.17(\mathrm{q}, 2 \mathrm{H}, \mathrm{J}=10.8 \mathrm{~Hz}), 7.70(\mathrm{~s}, 1 \mathrm{H}), 7.78(\mathrm{~s}, 1 \mathrm{H}), 9.51(\mathrm{~s}, 1 \mathrm{H})$. Elemental analysis. Calcd for $\mathrm{C}_{9} \mathrm{H}_{17} \mathrm{~N}_{3} \mathrm{O}_{2} \mathrm{~S}_{1} 2.1 \mathrm{H}_{2} \mathrm{O}: \mathrm{C}, 40.15 ; \mathrm{H}, 7.95 ; \mathrm{N}, 15.61 ; \mathrm{O}, 24.37 ; \mathrm{S}, 11.91$. Found: C,40.15; H, 8.35 ; N, 15.34.

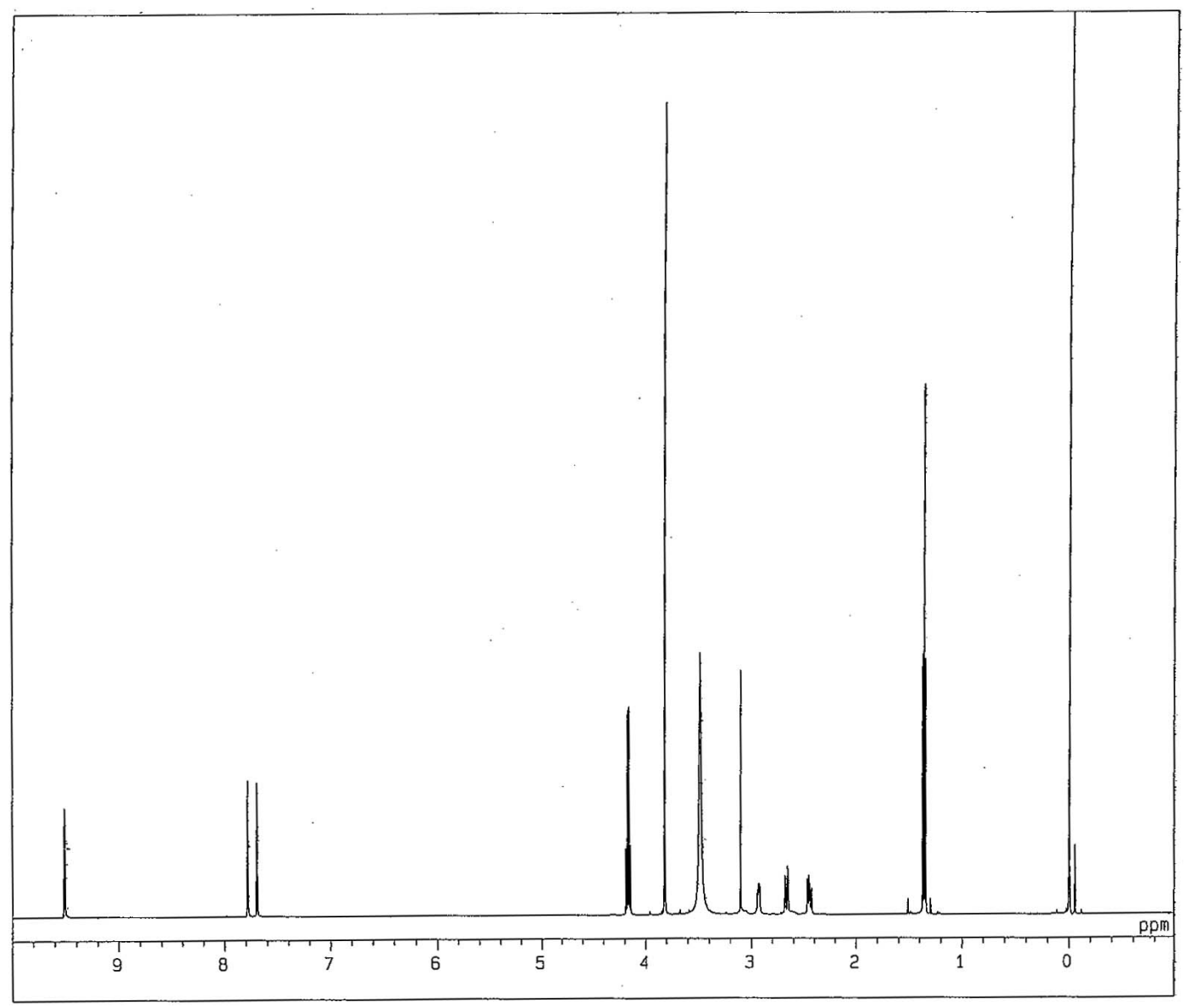


1-Ethyl-3-methylimidazolium 2-aminoglutaramic acid salt ([emim][Gln]). From 1.0g (5.2mmol) of [emim] $[\mathrm{Br}]$, and $0.8 \mathrm{~g}(5.5 \mathrm{mmol})$ of L-glutamine, $0.9 \mathrm{~g}$ of $[\mathrm{emim}][\mathrm{Gln}]$ was obtained (Yield : $66 \%) .{ }^{1} \mathrm{H}-$ NMR (DMSO, $\delta / p p m$ relative to TMS) : 1.35(t, 3H, J=7Hz), $1.43(\mathrm{~m}, 1 \mathrm{H}, \mathrm{J}=18 \mathrm{~Hz}), 1.66(\mathrm{~m}, 1 \mathrm{H}, \mathrm{J}=13 \mathrm{~Hz})$, $2.01(\mathrm{~m}, 2 \mathrm{H}, \mathrm{J}=23.8 \mathrm{~Hz}), 2.77(\mathrm{t}, 1 \mathrm{H}, \mathrm{J}=6.3 \mathrm{~Hz}), 3.82(\mathrm{~s}, 3 \mathrm{H}), 4.17(\mathrm{q}, 2 \mathrm{H}, \mathrm{J}=10.8 \mathrm{~Hz}), 6.55(\mathrm{~s}, 1 \mathrm{H}), 7.61(\mathrm{~s}$, 1H), 7.71(s, 1H), 7.79(s, 1H), 9.67(s, 1H).

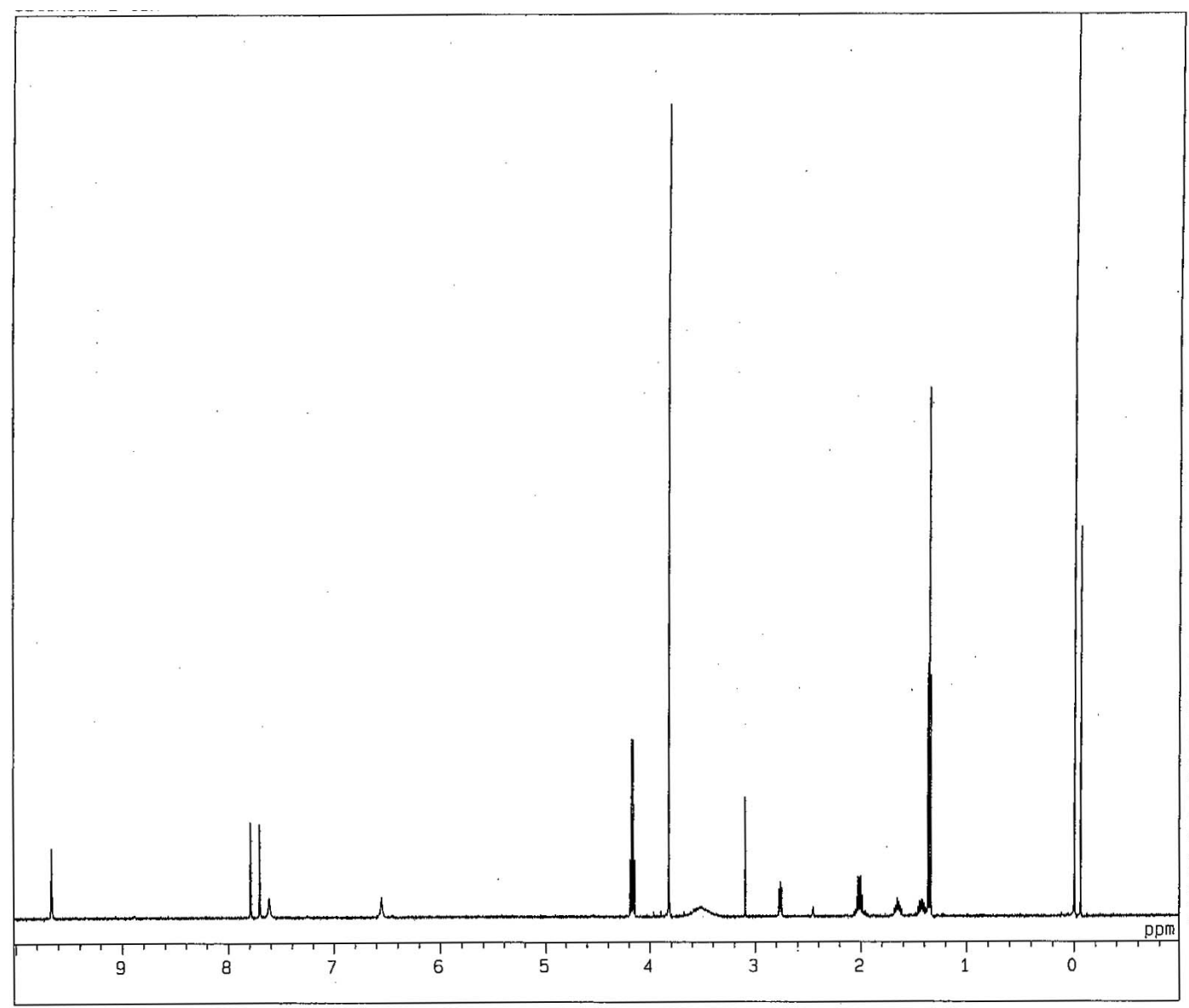


1-Ethyl-3-methylimidazolium 1-aminopropane-1,3-dicarboxylic acid salt ([emim][Glu]). From 1.0g $(5.2 \mathrm{mmol})$ of [emim] [Br] , and $0.9 \mathrm{~g}(6.8 \mathrm{mmol})$ of L-glutamic acid, $1.1 \mathrm{~g}$ of [emim][Glu] was obtained (Yield : 80\%). ${ }^{1} \mathrm{H}-\mathrm{NMR}$ (DMSO, $\delta / \mathrm{ppm}$ relative to TMS) : $1.35(\mathrm{t}, 3 \mathrm{H}, \mathrm{J}=7.5 \mathrm{~Hz}), 1.64(\mathrm{~m}, 1 \mathrm{H}, \mathrm{J}=17.3 \mathrm{~Hz}$ ), 1.77(m, 1H, J=14.5Hz), 2.07(m, 2H, J=32.8Hz), 3.03(q, 1H, J=5.8Hz), 3.11(s, 3H), 4.14(q, 2H, $\mathrm{J}=11.3 \mathrm{~Hz}), 7.66(\mathrm{t}, 1 \mathrm{H}, \mathrm{J}=1.5 \mathrm{~Hz}), 7.75(\mathrm{t}, 1 \mathrm{H}, \mathrm{J}=1.5 \mathrm{~Hz}), 9.25(\mathrm{~s}, 1 \mathrm{H})$. Elemental analysis. Calcd for $\mathrm{C}_{11} \mathrm{H}_{19} \mathrm{~N}_{3} \mathrm{O}_{4} 1.5 \mathrm{H}_{2} \mathrm{O}: \mathrm{C}, 46.46 ; \mathrm{H}, 7.81 ; \mathrm{N}, 14.78 ; \mathrm{O}, 30.95$. Found: C,46.48 ; H,7.83 ; N, 14.88 .

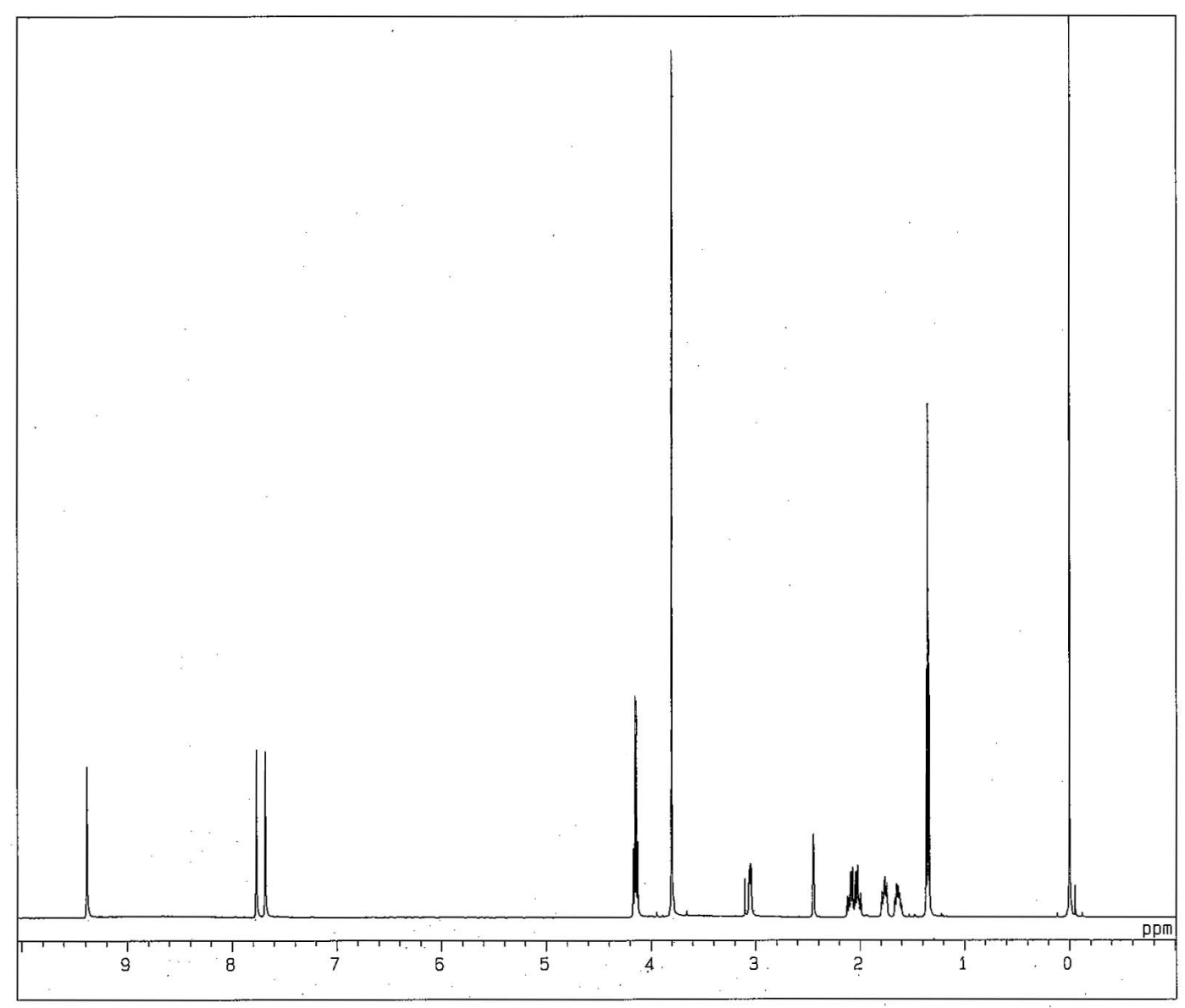


1-Ethyl-3-methylimidazolium aminoethanic acid salt ([emim][Gly]). From $1.0 \mathrm{~g}(5.2 \mathrm{mmol})$ of [emim] $[\mathrm{Br}]$, and $0.5 \mathrm{~g}(6.7 \mathrm{mmol})$ of L-glycin, $0.8 \mathrm{~g}$ of [emim] [Gly] was obtained (Yield : 82\%). ${ }^{1} \mathrm{H}-\mathrm{NMR}$ (DMSO, $\delta / p p m$ relative to TMS) : 1.35(t, 3H, J=7.5Hz), 2.67(s, 2H), 3.82(s, 3H), 4.17(q, 2H, J=11Hz), 7.73(s, $1 \mathrm{H}), 7.81(\mathrm{~s}, 1 \mathrm{H}), 9.78(\mathrm{~s}, 1 \mathrm{H})$. Elemental analysis. Calcd for $\mathrm{C}_{8} \mathrm{H}_{15} \mathrm{~N}_{3} \mathrm{O}_{2} \mathrm{H}_{2} \mathrm{O}$ : C, $49.46 ; \mathrm{H}, 8.32$; N, 21.63; O, 20.59. Found: C,49.18; H,8.25; N, 21.17.

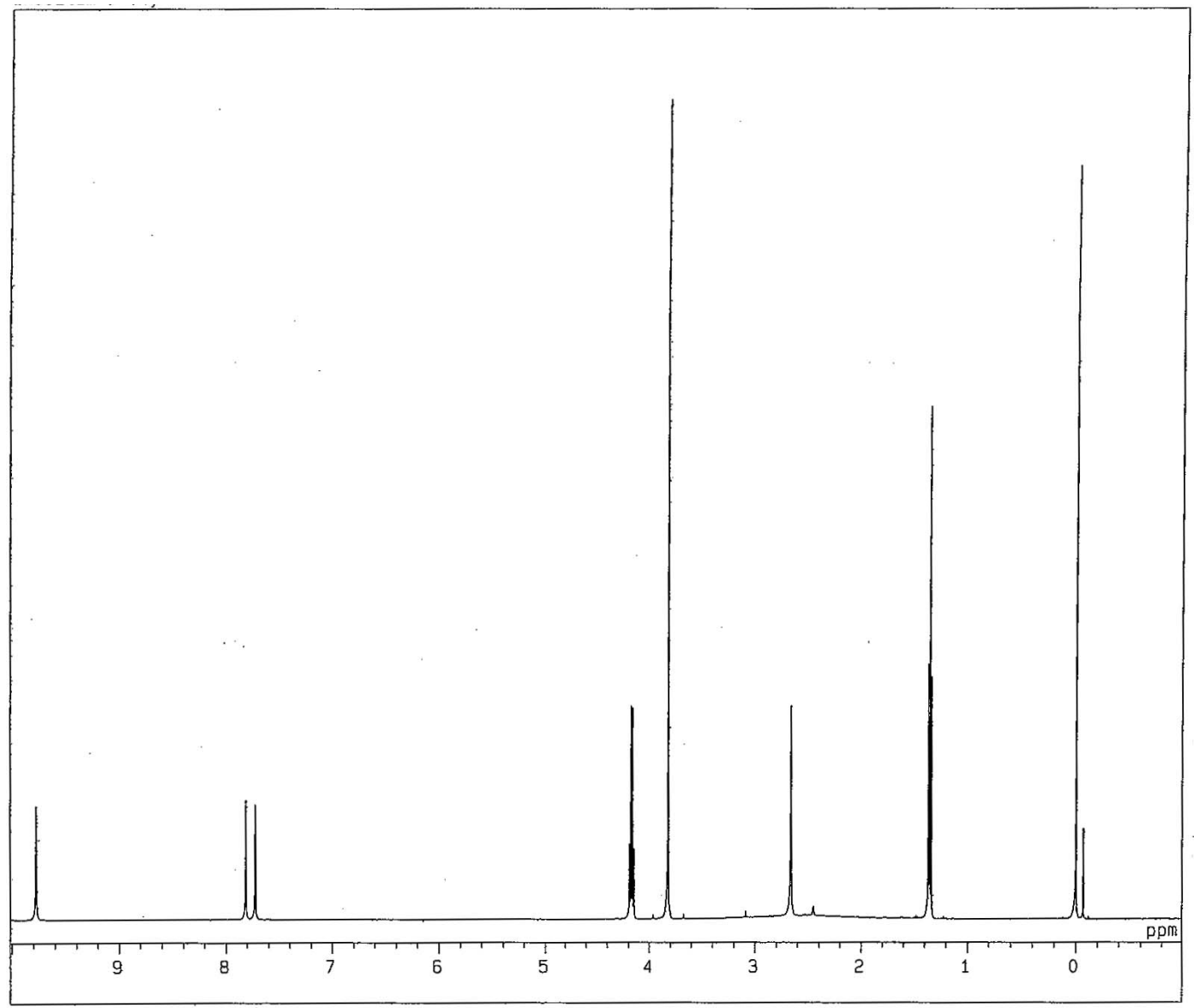


1-Ethyl-3-methylimidazolium $\alpha$-amino-4-imidazolepropionic acid salt ([emim][His]). From $1.0 \mathrm{~g}$ $(5.2 \mathrm{mmol})$ of [emim] $[\mathrm{Br}]$, and $1.0 \mathrm{~g}(6.4 \mathrm{mmol})$ of L-histidine, $1.1 \mathrm{~g}$ of [emim][His] was obtained (Yield : 78\%). ${ }^{1} \mathrm{H}-\mathrm{NMR}$ (DMSO, $\delta / \mathrm{ppm}$ relative to TMS) : 1.34(t, 3H, J=7Hz), 2.36(q, 1H, J=11Hz), 2.82(q, 1H, $\mathrm{J}=9.3 \mathrm{~Hz}), 2.99(\mathrm{t}, 1 \mathrm{H}, \mathrm{J}=6 \mathrm{~Hz}), 3.80(\mathrm{~s}, 3 \mathrm{H}), 4.14(\mathrm{q}, 2 \mathrm{H}, \mathrm{J}=10.8 \mathrm{~Hz}), 6.58(\mathrm{~s}, 1 \mathrm{H}), 7.35(\mathrm{~s}, 1 \mathrm{H}), 7.68(\mathrm{~s}, 1 \mathrm{H})$, 7.76(s, 1H), 9.53(s, 1H).

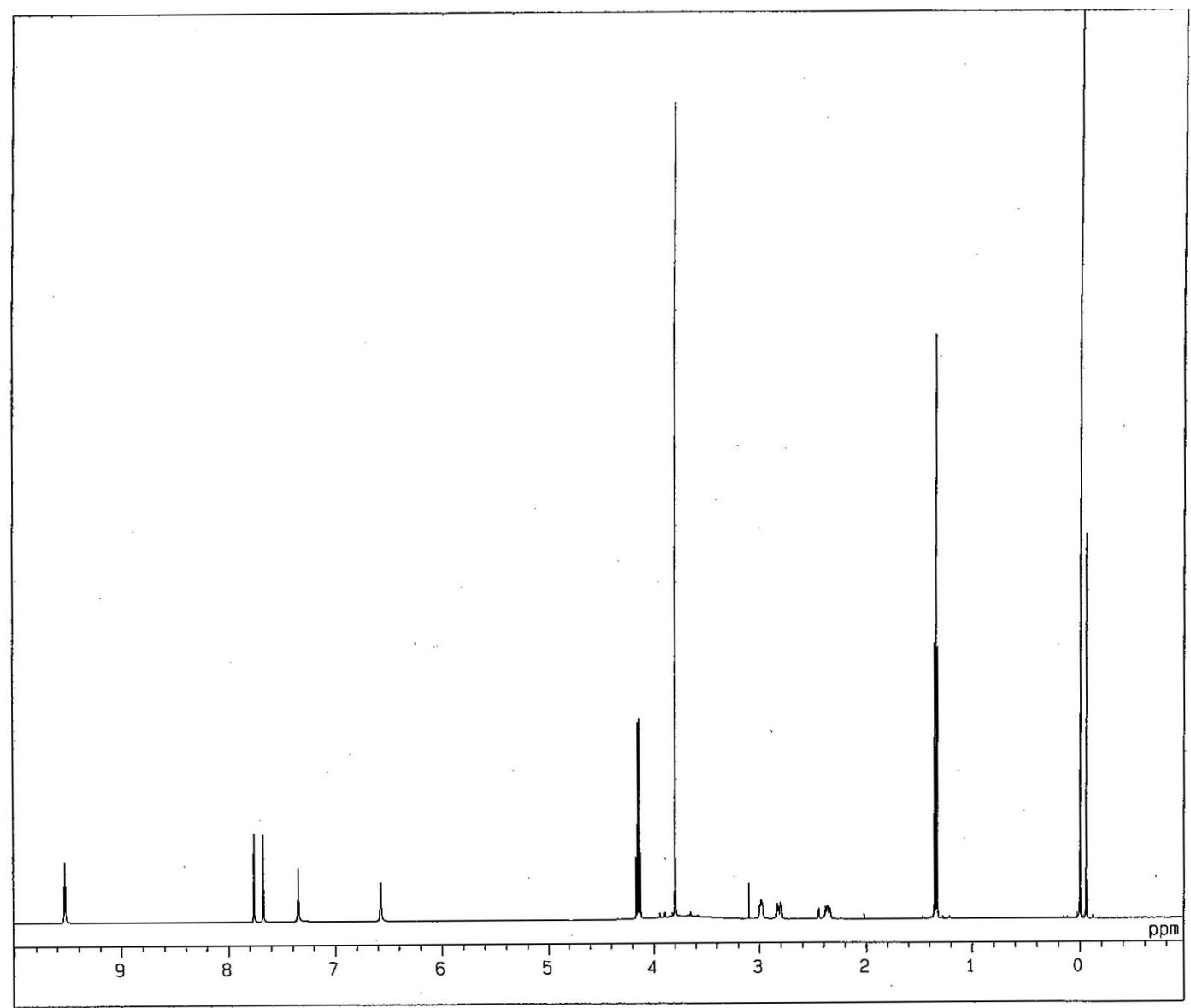


1-Ethyl-3-methylimidazolium 2-amino-3-methylvaleric acid salt ([emim] [Ile]). From 1.0g (5.2mmol) of [emim] [Br] , and $0.9 \mathrm{~g}(6.1 \mathrm{mmol})$ of L-isoleucine, $1.0 \mathrm{~g}$ of [emim][Ile] was obtained (Yield : $82 \%) .{ }^{1} \mathrm{H}-$ NMR (DMSO, $\delta / p p m$ relative to TMS) : $0.77(\mathrm{~m}, 6 \mathrm{H}, \mathrm{J}=12.3 \mathrm{~Hz}), 0.96(\mathrm{~m}, 1 \mathrm{H}, \mathrm{J}=22 \mathrm{~Hz}), 1.30(\mathrm{~m}, 1 \mathrm{H}$, $\mathrm{J}=13.5 \mathrm{~Hz}), 1.36(\mathrm{t}, 3 \mathrm{H}, \mathrm{J}=7.5 \mathrm{~Hz}), 1.53(\mathrm{~m}, 1 \mathrm{H}, \mathrm{J}=22.3 \mathrm{~Hz}), 2.65(\mathrm{~d}, 1 \mathrm{H}, \mathrm{J}=2.3 \mathrm{~Hz}), 3.83(\mathrm{~s}, 3 \mathrm{H}), 4.18(\mathrm{q}, 2 \mathrm{H}$, $\mathrm{J}=11 \mathrm{~Hz}), 7.72(\mathrm{~s}, 1 \mathrm{H}), 7.81(\mathrm{~s}, 1 \mathrm{H}), 9.80(\mathrm{~s}, 1 \mathrm{H})$. Elemental analysis. Calcd for $\mathrm{C}_{12} \mathrm{H}_{23} \mathrm{~N}_{3} \mathrm{O}_{2} 2 \mathrm{H}_{2} \mathrm{O}: \mathrm{C}$, 51.95 ; H, 9.83; N, 15.15; O, 23.07. Found: C,51.88 ; H,9.68 ; N, 14.99.

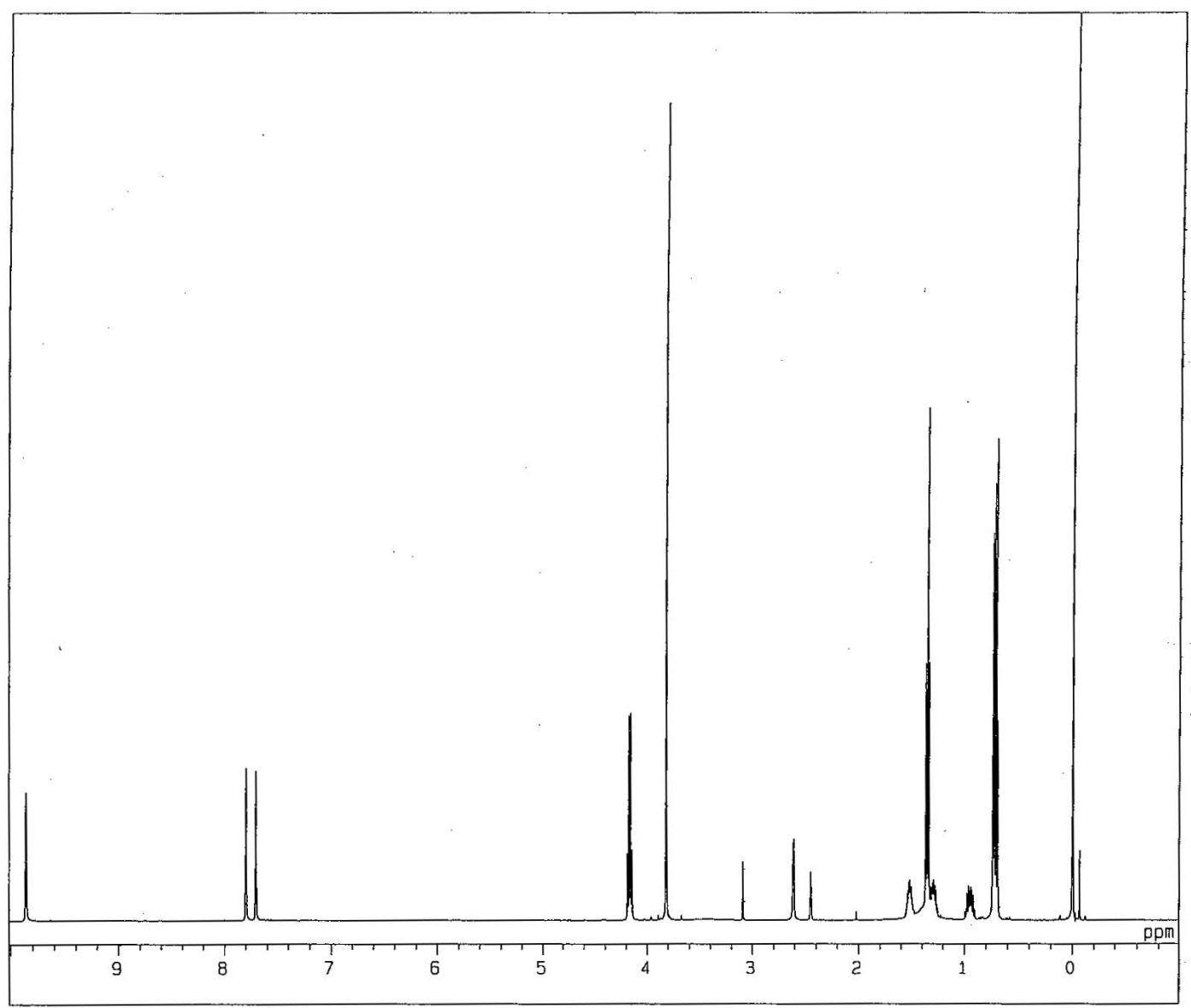


1-Ethyl-3-methylimidazolium 2-amino-4-methylvaleric acid salt ([emim][Leu]). From 1.0g

(5.2mmol) of [emim] [Br] and $0.8 \mathrm{~g}(6.1 \mathrm{mmol})$ of L-isoleucine, $0.8 \mathrm{~g}$ of [emim][Ile] was obtained (Yield : 80\%). ${ }^{1} \mathrm{H}-\mathrm{NMR}$ (DMSO, $\delta / \mathrm{ppm}$ relative to TMS) : $0.75(\mathrm{~d}, 3 \mathrm{H}, \mathrm{J}=3.3 \mathrm{~Hz}), 0.79(\mathrm{~d}, 3 \mathrm{H}, \mathrm{J}=3.5 \mathrm{~Hz}), 1.01(\mathrm{~m}$, $1 \mathrm{H}), 1.36(\mathrm{t}, 3 \mathrm{H}, \mathrm{J}=7 \mathrm{~Hz}), 1.65(\mathrm{~m}, 1 \mathrm{H}, \mathrm{J}=20.5 \mathrm{~Hz}), 2.75(\mathrm{q}, 1 \mathrm{H}, \mathrm{J}=7 \mathrm{~Hz}), 3.83(\mathrm{~s}, 3 \mathrm{H}), 4.18(\mathrm{q}, 2 \mathrm{H}, \mathrm{J}=10.8 \mathrm{~Hz})$, 7.72(s,1H), 7.82(s, $1 \mathrm{H}), 9.79(\mathrm{~s}, 1 \mathrm{H})$. Elemental analysis. Calcd for $\mathrm{C}_{12} \mathrm{H}_{23} \mathrm{~N}_{3} \mathrm{O}_{2} 4 \mathrm{H}_{2} \mathrm{O}: \mathrm{C}, 44.69 ; \mathrm{H}$, 10.02; N, 13.03; O, 32.25. Found: C,44.66; H,9.61; N, 12.81.

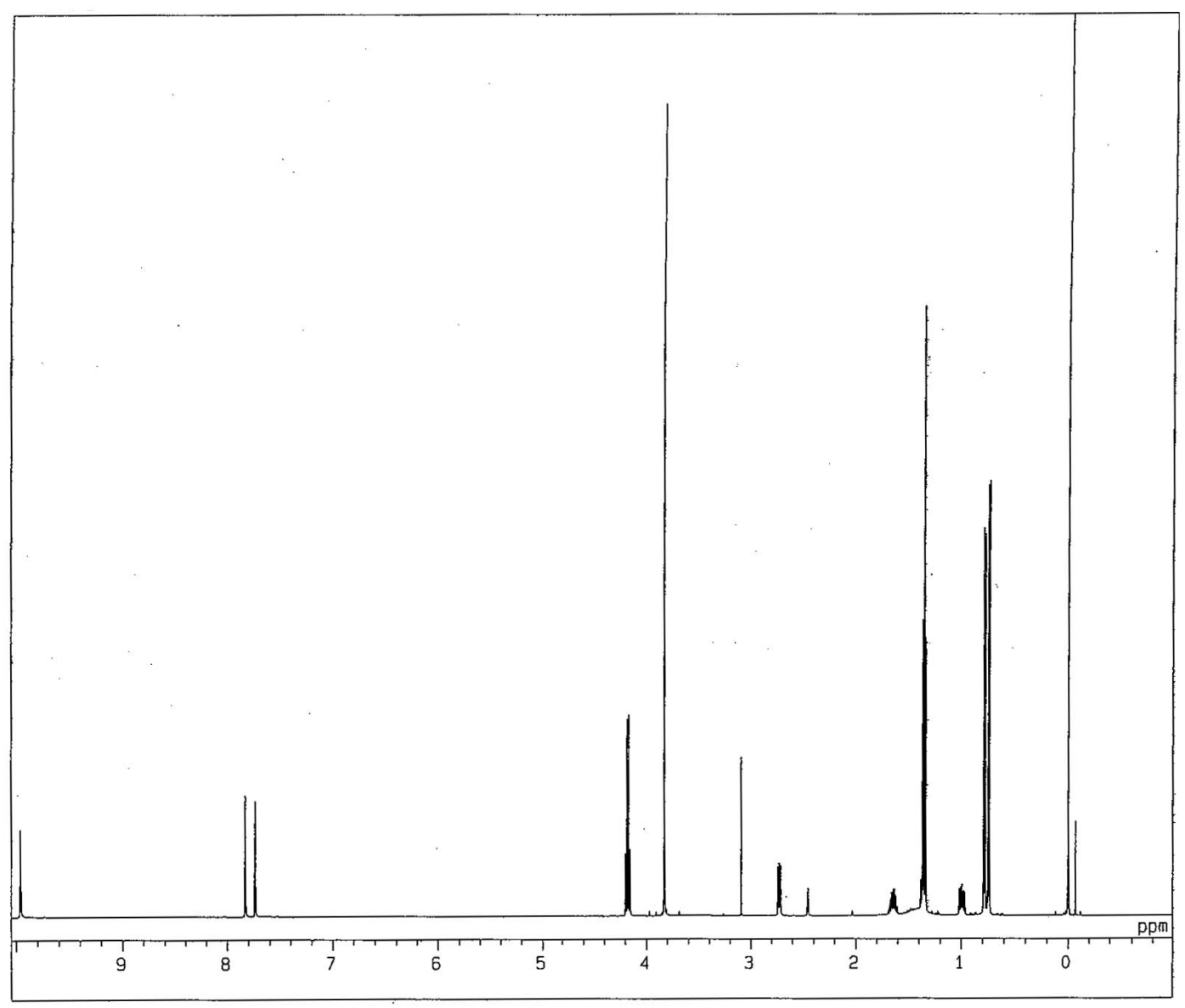


1-Ethyl-3-methylimidazolium $\boldsymbol{\alpha}, \boldsymbol{\varepsilon}$-diaminocaproic acid salt ([emim][Lys]). From 1.0g (5.2mmol) of [emim] $[\mathrm{Br}]$, and $0.9 \mathrm{~g}(6.2 \mathrm{mmol})$ of L-isoleucine, $1.0 \mathrm{~g}$ of [emim] [Lys] was obtained (Yield : 78\%). ${ }^{1} \mathrm{H}-$ NMR (DMSO, $\delta / p p m$ relative to TMS) : $1.22(\mathrm{~m}, 5 \mathrm{H}, \mathrm{J}=13.5 \mathrm{~Hz}), 1.36(\mathrm{t}, 3 \mathrm{H}, \mathrm{J}=7.5 \mathrm{~Hz}), 1.48(\mathrm{~m}, 1 \mathrm{H}$, $\mathrm{J}=13 \mathrm{~Hz}), 2.42(\mathrm{~d}, 2 \mathrm{H}, \mathrm{J}=6.5 \mathrm{~Hz}), 2.73(\mathrm{t}, 1 \mathrm{H}, \mathrm{J}=7.0 \mathrm{~Hz}), 3.82(\mathrm{~s}, 3 \mathrm{H}), 4.17(\mathrm{q}, 2 \mathrm{H}, \mathrm{J}=11.3 \mathrm{~Hz}), 7.70(\mathrm{t}, 1 \mathrm{H}$, $\mathrm{J}=1.5 \mathrm{~Hz}), 7.79(\mathrm{t}, 1 \mathrm{H}, \mathrm{J}=1.5 \mathrm{~Hz}), 9.67(\mathrm{~s}, 1 \mathrm{H})$. Elemental analysis. Calcd for $\mathrm{C}_{12} \mathrm{H}_{24} \mathrm{~N}_{4} \mathrm{O}_{2} \mathrm{H}_{2} \mathrm{O}: \mathrm{C}, 52.52$; H, 9.57; N, 20.42; O, 17.49. Found: C,52.95; H,9.47 ; N, 20.16.

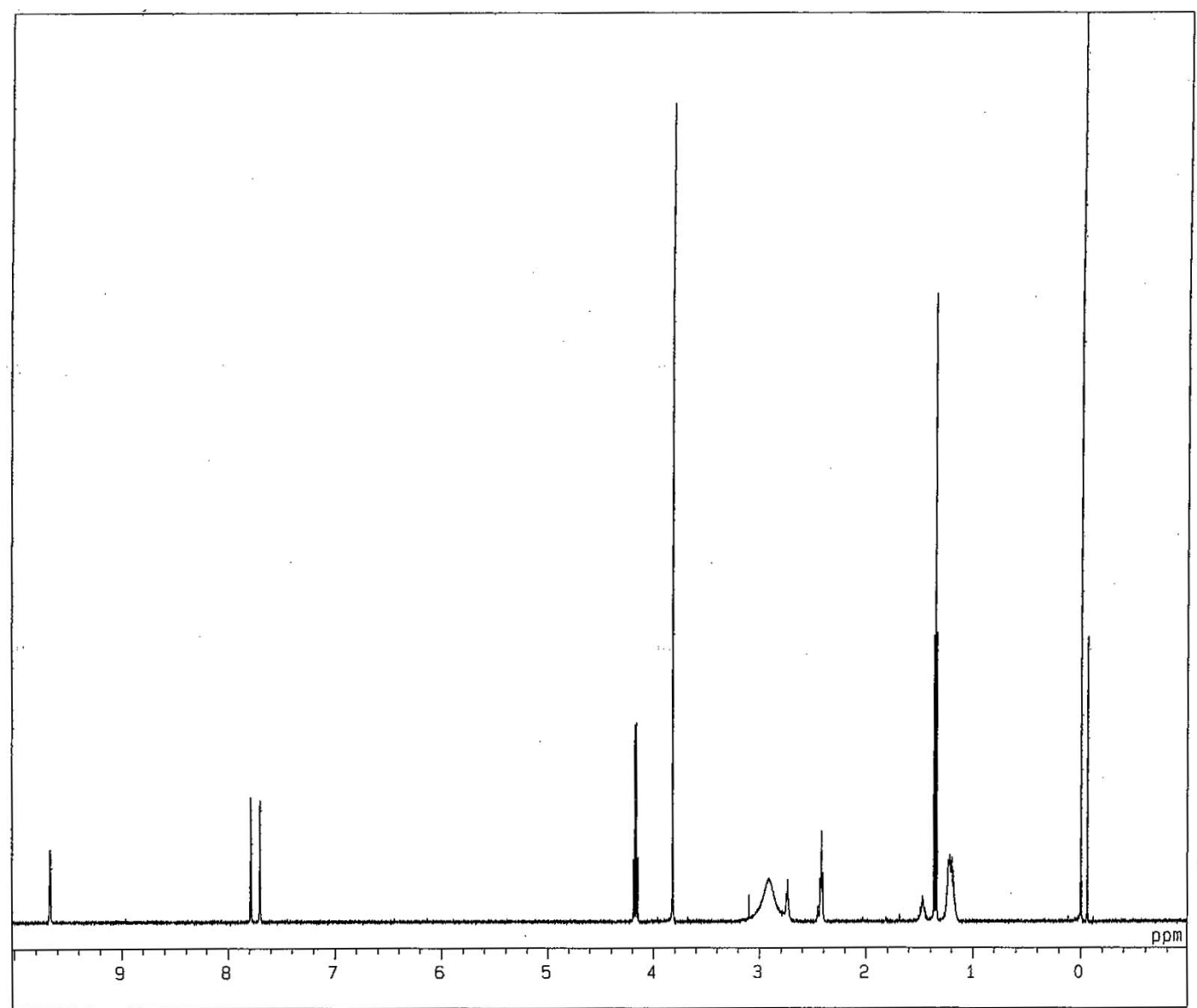


1-Ethyl-3-methylimidazolium 2-amino-4-(methylthio)butyric acid salt ([emim][Met]). From 1.0g (5.2mmol) of [emim] [Br] and $0.9 \mathrm{~g}(6.0 \mathrm{mmol})$ of $\mathrm{L}-$ methionine, $1.1 \mathrm{~g}$ of [emim][Met] was obtained (Yield : 78\%). ${ }^{1} \mathrm{H}-\mathrm{NMR}$ (DMSO, $\delta / \mathrm{ppm}$ relative to TMS) : $1.36(\mathrm{t}, 3 \mathrm{H}, \mathrm{J}=7.5 \mathrm{~Hz}), 1.42(\mathrm{~m}, 1 \mathrm{H}, \mathrm{J}=24.5 \mathrm{~Hz}$ ), 1.74(m, 1H, J=16.5Hz), 1.94(s, 1H), 2.42(t, 2H, J=8.5Hz), 2.82(q, 1H, J=5.75Hz), 3.82(s, 3H), 4.17(q, $2 \mathrm{H}, \mathrm{J}=11.3 \mathrm{~Hz}), 7.71(\mathrm{~s}, 1 \mathrm{H}), 7.80(\mathrm{~s}, 1 \mathrm{H}), 9.69(\mathrm{~s}, 1 \mathrm{H})$. Elemental analysis. Calcd for $\mathrm{C}_{11} \mathrm{H}_{21} \mathrm{~N}_{3} \mathrm{O}_{2} \mathrm{~S}$ 2.3H $\mathrm{H}_{2} \mathrm{O}: \mathrm{C}, 43.91 ; \mathrm{H}, 8.59 ; \mathrm{N}, 13.97 ; \mathrm{O}, 22.87 ; \mathrm{S}, 10.66$. Found: C,43.87 ; H,8.51 ; N, 13.86.

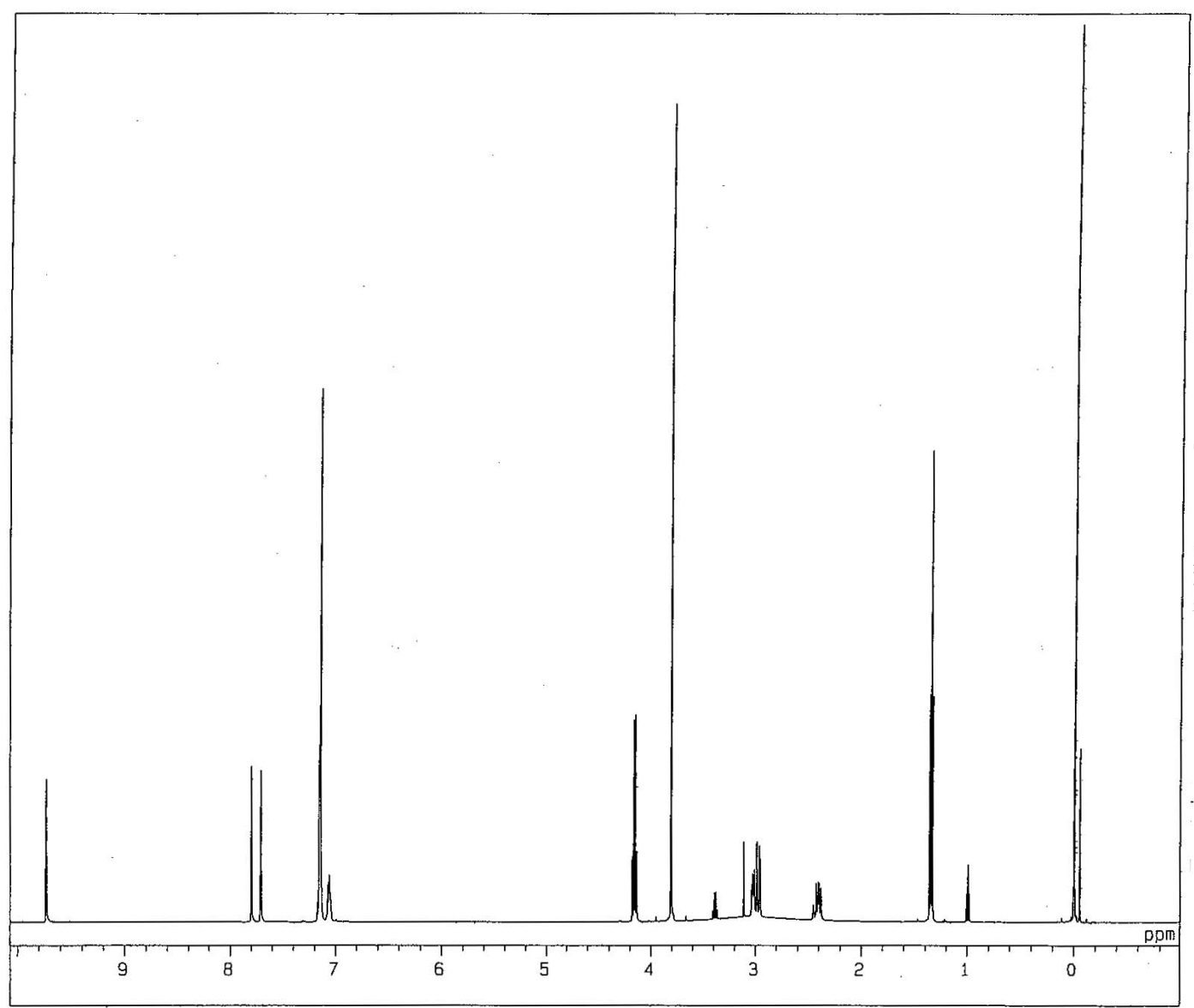


1-Ethyl-3-methylimidazolium $\alpha$-aminohydrocinnamic acid salt ([emim] [Phe]). From $1.0 \mathrm{~g}(5.2 \mathrm{mmol})$ of [emim][Br] and $1.0 \mathrm{~g}(6.1 \mathrm{mmol})$ of L-phenylalanine, $1.2 \mathrm{~g}$ of [emim][Phe] was obtained (Yield : $83 \%)$. ${ }^{1} \mathrm{H}-\mathrm{NMR}$ (DMSO, $\delta / \mathrm{ppm}$ relative to TMS) : 1.34(t, 3H, J=7Hz), 2.40(q, 1H, J=11Hz), 2.99(m, 2H, $\mathrm{J}=19.8 \mathrm{~Hz}), 4.13(\mathrm{~s}, 3 \mathrm{H}), 4.16(\mathrm{q}, 2 \mathrm{H}, \mathrm{J}=11.3 \mathrm{~Hz}), 7.06(\mathrm{~m}, 1 \mathrm{H}, \mathrm{J}=11.25 \mathrm{~Hz}), 7.15(\mathrm{t}, 4 \mathrm{H}, \mathrm{J}=3 \mathrm{~Hz}), 7.71(\mathrm{~s}, 1 \mathrm{H})$, 7.80(s, $1 \mathrm{H}), 9.74(\mathrm{~s}, 1 \mathrm{H})$. Elemental analysis. Calcd for $\mathrm{C}_{15} \mathrm{H}_{21} \mathrm{~N}_{3} \mathrm{O}_{2} 1.7 \mathrm{H}_{2} \mathrm{O}: \mathrm{C}, 58.87 ; \mathrm{H}, 8.05 ; \mathrm{N}, 13.73$; O, 19.34. Found: C,58.36; H,8.01; N, 13.58.

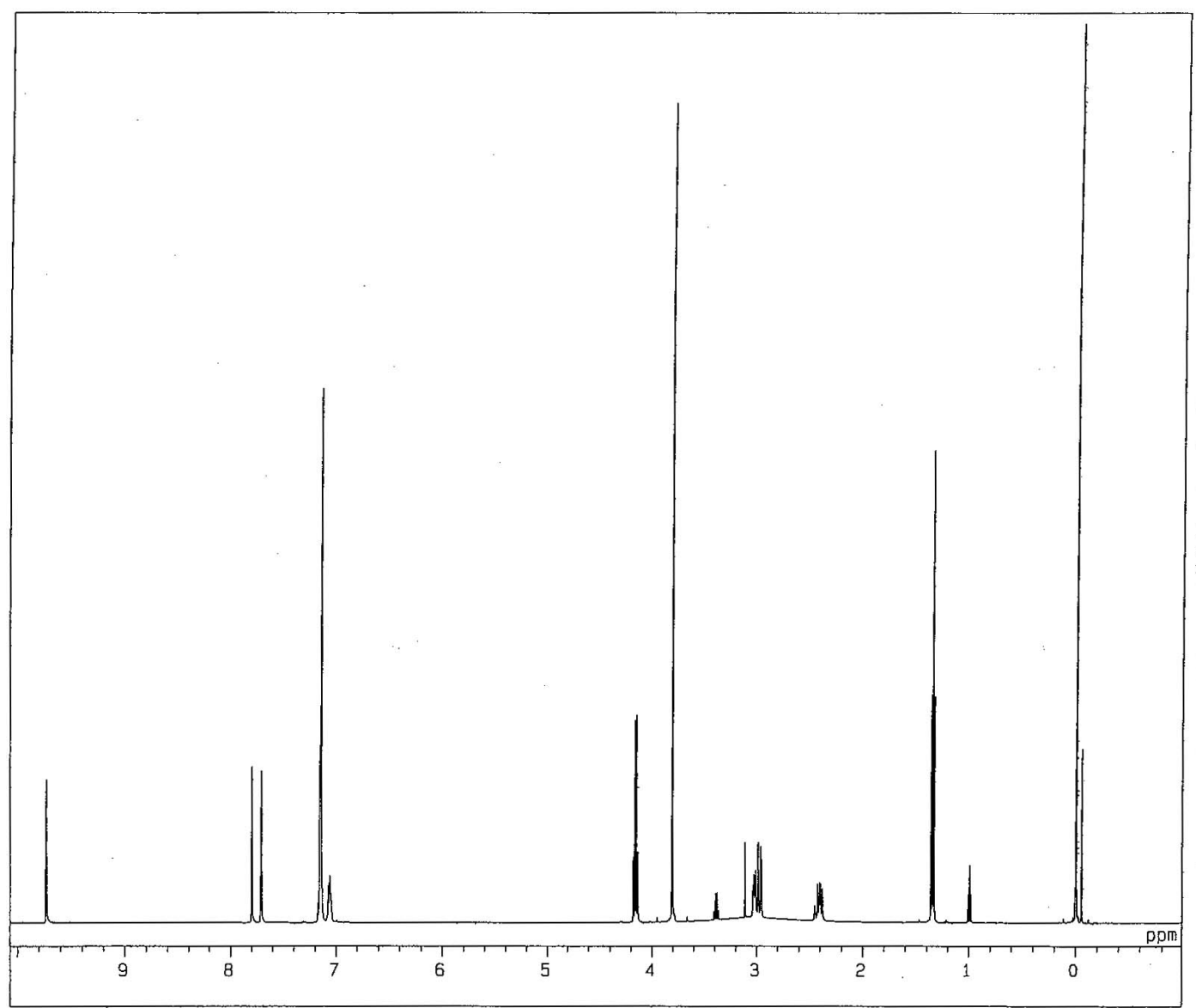


1-Ethyl-3-methylimidazolium (S)-2-pyrrolidinecarboxylic acid salt ([emim][Pro]). From 1.0g (5.2mmol) of [emim] $[\mathrm{Br}]$, and $1.0 \mathrm{~g}(8.7 \mathrm{mmol})$ of L-proline, $1.1 \mathrm{~g}$ of [emim][Pro] was obtained (Yield : 83\%). ${ }^{1} \mathrm{H}-\mathrm{NMR}$ (DMSO, $\delta / \mathrm{ppm}$ relative to TMS): $1.36(\mathrm{t}, 3 \mathrm{H}, \mathrm{J}=7.5 \mathrm{~Hz}), 1.42(\mathrm{~m}, 1 \mathrm{H}, \mathrm{J}=17 \mathrm{~Hz}), 1.51(\mathrm{~m}$, $1 \mathrm{H}, \mathrm{J}=19 \mathrm{~Hz}), 1.62(\mathrm{broad}, 1 \mathrm{H}), 1.77(\mathrm{~m}, 1 \mathrm{H}, \mathrm{J}=17.8 \mathrm{~Hz}), 2.60($ broad, $1 \mathrm{H}), 2,92(\mathrm{~m}, 1 \mathrm{H}, \mathrm{J}=11.3 \mathrm{~Hz})$, 3.18(broad, 1H), 3.82(s, 3H), 4.16(q, 2H, J=11.3Hz), 7.70(s, 1H), 7.79(s, 1H), 9.53(s, 1H). Elemental analysis. Calcd for $\mathrm{C}_{11} \mathrm{H}_{19} \mathrm{~N}_{3} \mathrm{O}_{2} 2.25 \mathrm{H}_{2} \mathrm{O}: \mathrm{C}, 49.69 ; \mathrm{H}, 8.92 ; \mathrm{N}, 15.81 ; \mathrm{O}, 25.58$. Found: $\mathrm{C}, 50.16 ; \mathrm{H}, 8.85$; $\mathrm{N}, 15.32$.

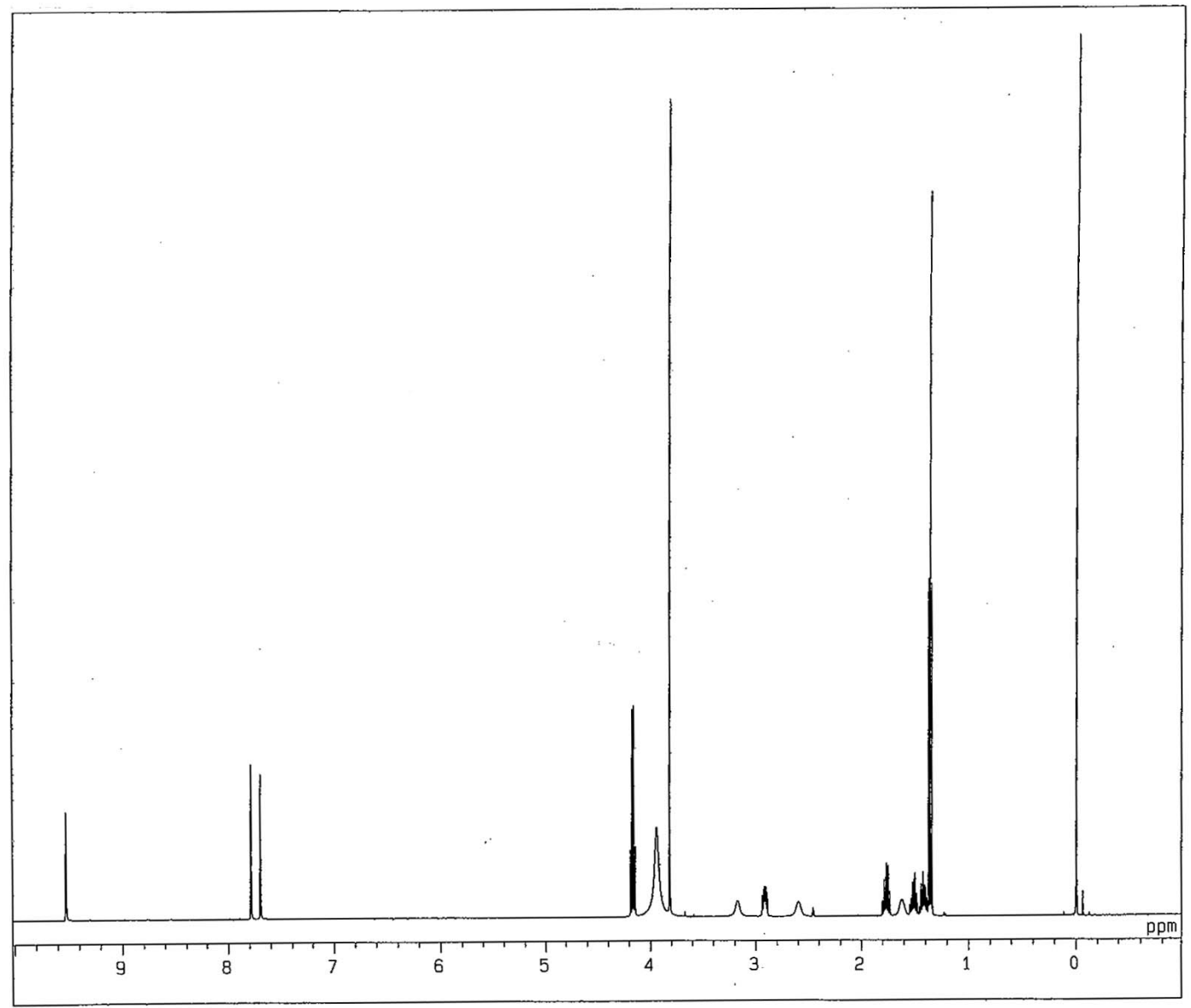


1-Ethyl-3-methylimidazolium 2-amino-3-hydroxypropionic acid salt ([emim][Ser]). From 1.0g $(5.2 \mathrm{mmol})$ of [emim] [Br] and $0.7 \mathrm{~g}(6.7 \mathrm{mmol})$ of L-serine, $0.9 \mathrm{~g}$ of [emim][Ser] was obtained (Yield : 79\%). ${ }^{1} \mathrm{H}-\mathrm{NMR}$ (DMSO, $\delta / \mathrm{ppm}$ relative to TMS) : 1.36(t, 3H, J=7Hz), 2.83(t, 1H, J=7Hz), 3.23(m, 2H, $\mathrm{J}=15.5 \mathrm{~Hz}), 3.81(\mathrm{~s}, 3 \mathrm{H}), 4.16(\mathrm{q}, 2 \mathrm{H}, \mathrm{J}=11 \mathrm{~Hz}), 7.69(\mathrm{~s}, 1 \mathrm{H}), 7.78(\mathrm{~s}, 1 \mathrm{H}), 9.47(\mathrm{~s}, 1 \mathrm{H})$. Elemental analysis. Calcd for $\mathrm{C}_{9} \mathrm{H}_{17} \mathrm{~N}_{3} \mathrm{O}_{3} \mathrm{H}_{2} \mathrm{O}: \mathrm{C}, 46.33 ; \mathrm{H}, 8.23 ; \mathrm{N}, 18.00 ; \mathrm{O}, 27.43$. Found: $\mathrm{C}, 46.37 ; \mathrm{H}, 8.22 ; \mathrm{N}, 17.71$.

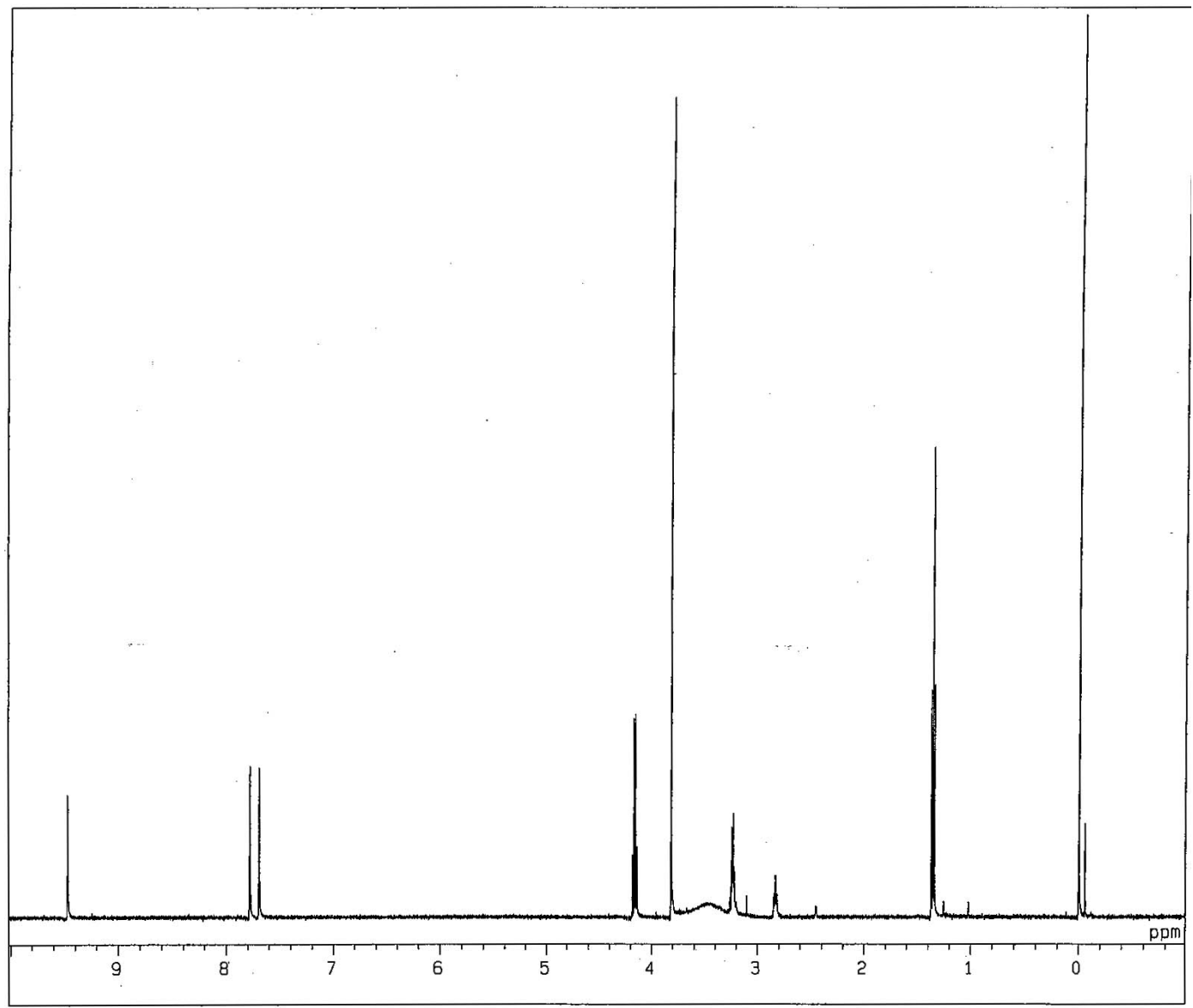


1-Ethyl-3-methylimidazolium 2-amino-3-hydroxybutyric acid salt ([emim][Thr]). From 1.0g (5.2mmol) of [emim] [Br] and $0.7 \mathrm{~g}(5.9 \mathrm{mmol})$ of L-threonine, $1.0 \mathrm{~g}$ of [emim][Thr] was obtained (Yield : 84\%). ${ }^{1} \mathrm{H}-\mathrm{NMR}$ (DMSO, $\delta / \mathrm{ppm}$ relative to TMS) : 0.86(d, 3H, J=3Hz), 1.36(t, 3H, J=7Hz), 2.82(d, $1 \mathrm{H}$, $\mathrm{J}=2.5 \mathrm{~Hz}), 3.44(\mathrm{~m}, 1 \mathrm{H}, \mathrm{J}=12 \mathrm{~Hz}), 3.82(\mathrm{~s}, 3 \mathrm{H}), 4.17(\mathrm{q}, 2 \mathrm{H}, \mathrm{J}=10.8 \mathrm{~Hz}), 7.70(\mathrm{~s}, 1 \mathrm{H}), 7.79(\mathrm{~s}, 1 \mathrm{H}), 9.52(\mathrm{~s}$, 1H). Elemental analysis. Calcd for $\mathrm{C}_{10} \mathrm{H}_{19} \mathrm{~N}_{3} \mathrm{O}_{3} 1.4 \mathrm{H}_{2} \mathrm{O}: \mathrm{C}, 47.18 ; \mathrm{H}, 8.65 ; \mathrm{N}, 16.51 ; \mathrm{O}, 27.66$. Found: $\mathrm{C}, 47.31 ; \mathrm{H}, 8.62 ; \mathrm{N}, 16.44$.

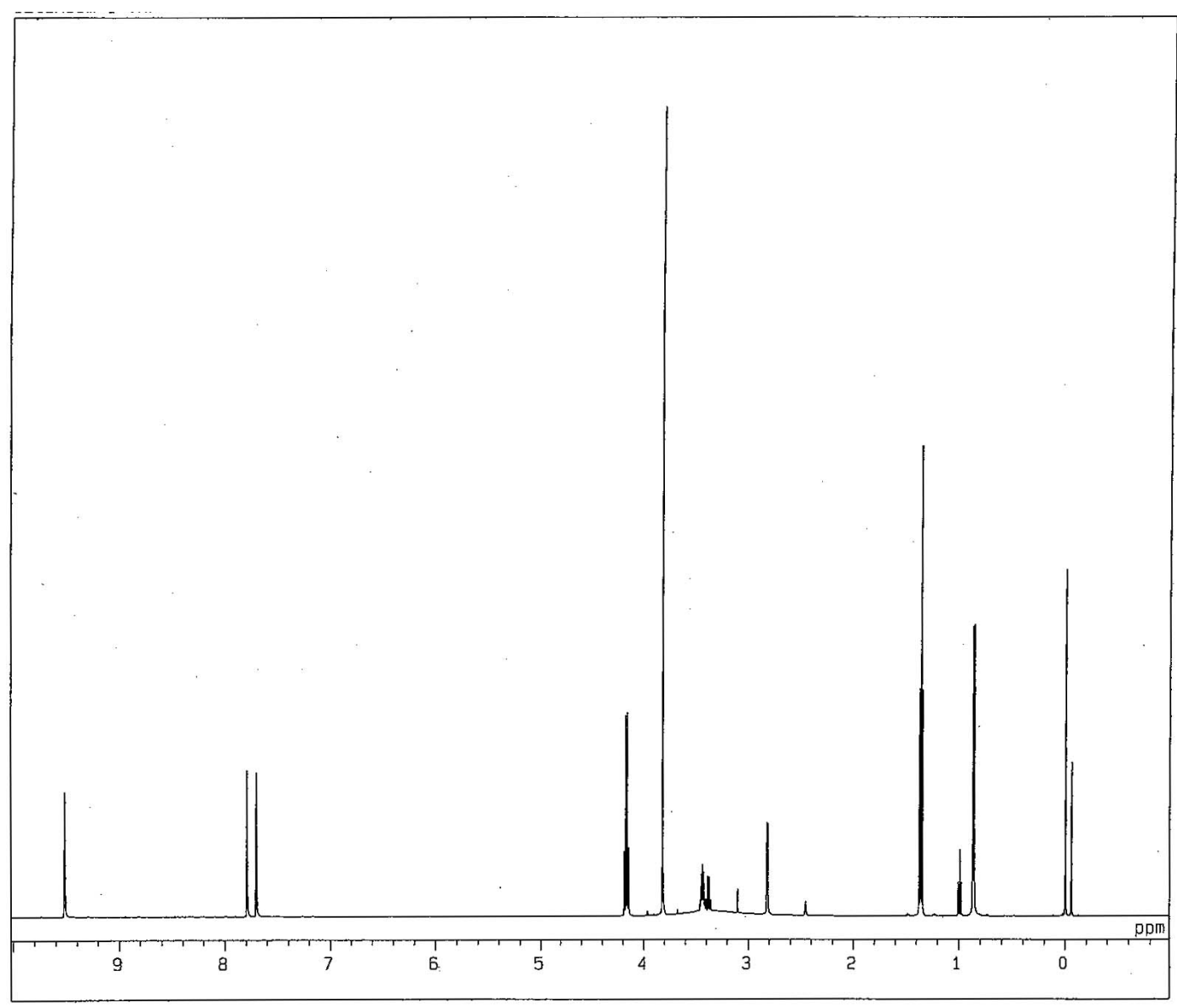


1-Ethyl-3-methylimidazolium (S)- $\alpha$-amino-1H-indole-3-propanoic acid salt ([emim][Trp]). From $1.5 \mathrm{~g}(7.9 \mathrm{mmol})$ of [emim] $[\mathrm{Br}]$, and $1.8 \mathrm{~g}(8.8 \mathrm{mmol})$ of L-tryptophan, $1.0 \mathrm{~g}$ of [emim][Trp] was obtained (Yield : 82\%). ${ }^{1} \mathrm{H}-\mathrm{NMR}$ (DMSO, $\delta / \mathrm{ppm}$ relative to TMS) : 1.32(t, 3H, J=7.5Hz), 2.52(q, 1H, J=12Hz), 3.08(d, 2H, J=3.5Hz), 3.77(s, 3H), 4.11(q, 2H, J=11Hz), 6.83(t, 1H, J=7Hz), 6.93(t, 1H, J=7.5Hz), 7.06(s, 1H), 7.27(d, 2H, J=4Hz), 7.45(d, 1H, J=4Hz), 7.64(s, 1H), 7.72(s, 1H), 9.5(s, 1H), 11.43(s, 1H).

Elemental analysis. Calcd for $\mathrm{C}_{17} \mathrm{H}_{22} \mathrm{~N}_{4} \mathrm{O}_{2}$ 1.4H $\mathrm{H}_{2} \mathrm{O}: \mathrm{C}, 60.11 ; \mathrm{H}, 7.37 ; \mathrm{N}, 16.50 ; \mathrm{O}, 16.02$. Found: C,60.35 ; H,7.53 ; N, 16.22.

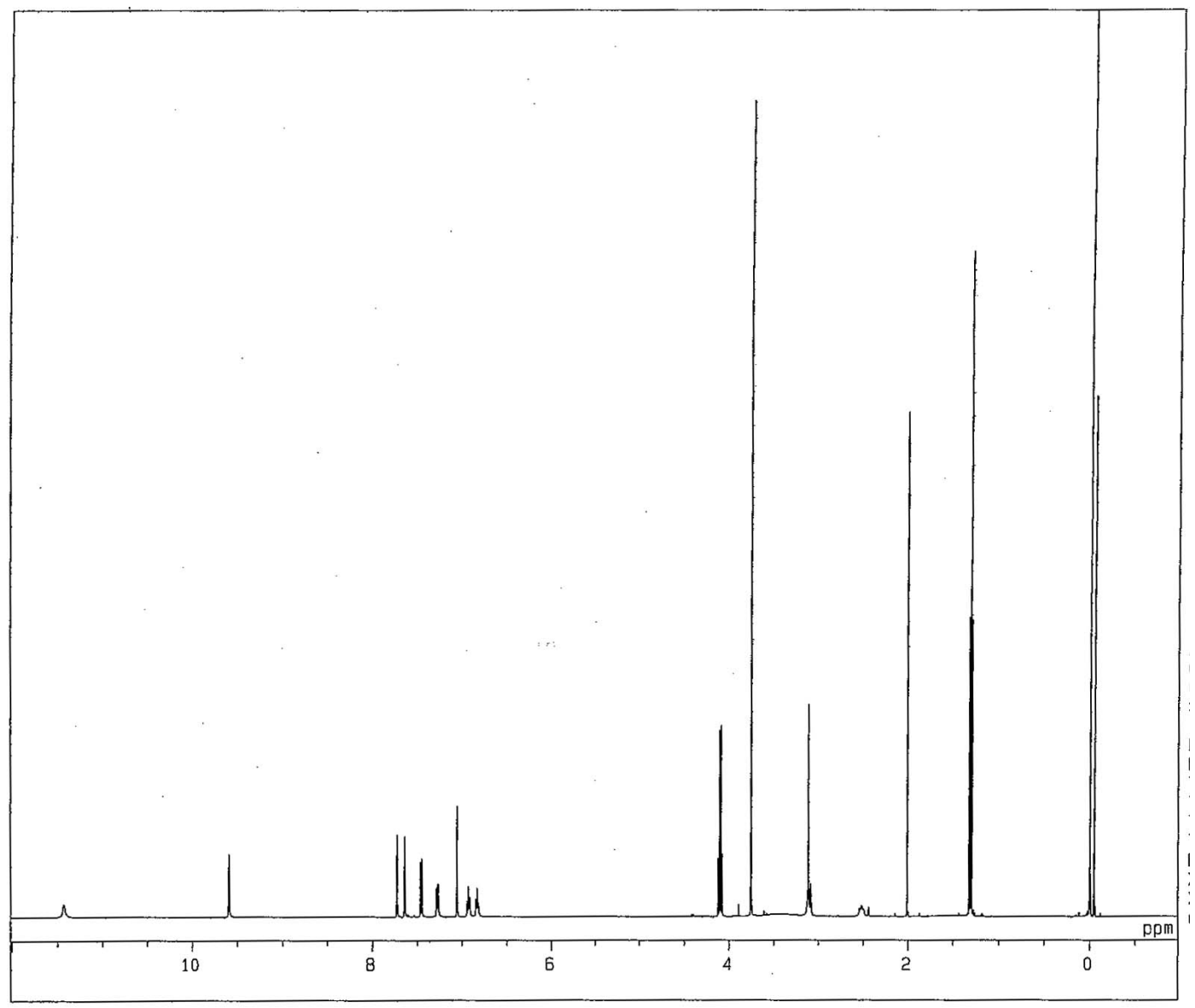


1-Ethyl-3-methylimidazolium $\alpha$-amino- $\rho$-hydroxyhydrocinnamic acid salt ([emim][Tyr]). From $2.0 \mathrm{~g}(10.5 \mathrm{mmol})$ of [emim][Br] and $2.2 \mathrm{~g}(12.1 \mathrm{mmol})$ of L-tyrosine, $2.1 \mathrm{~g}$ of [emim][Tyr] was obtained (Yield : 70\%). ${ }^{1} \mathrm{H}-\mathrm{NMR}$ (DMSO, $\delta / \mathrm{ppm}$ relative to TMS) :1.34(t, 3H, J=7Hz), 2.11(t, 1H, J=11.5Hz), $2.80(\mathrm{~m}, 2 \mathrm{H}, \mathrm{J}=25.7 \mathrm{~Hz}), 3.78(\mathrm{~s}, 3 \mathrm{H}), 4.13(\mathrm{q}, 2 \mathrm{H}, \mathrm{J}=10.8 \mathrm{~Hz}), 6.38(\mathrm{~d}, 2 \mathrm{H}, \mathrm{J}=4.3 \mathrm{~Hz}), 6.70(\mathrm{~d}, 2 \mathrm{H}, \mathrm{J}=4.3 \mathrm{H})$, 7.65(s, 1H), 7.74(s, 1H), 9.48(s, 1H).

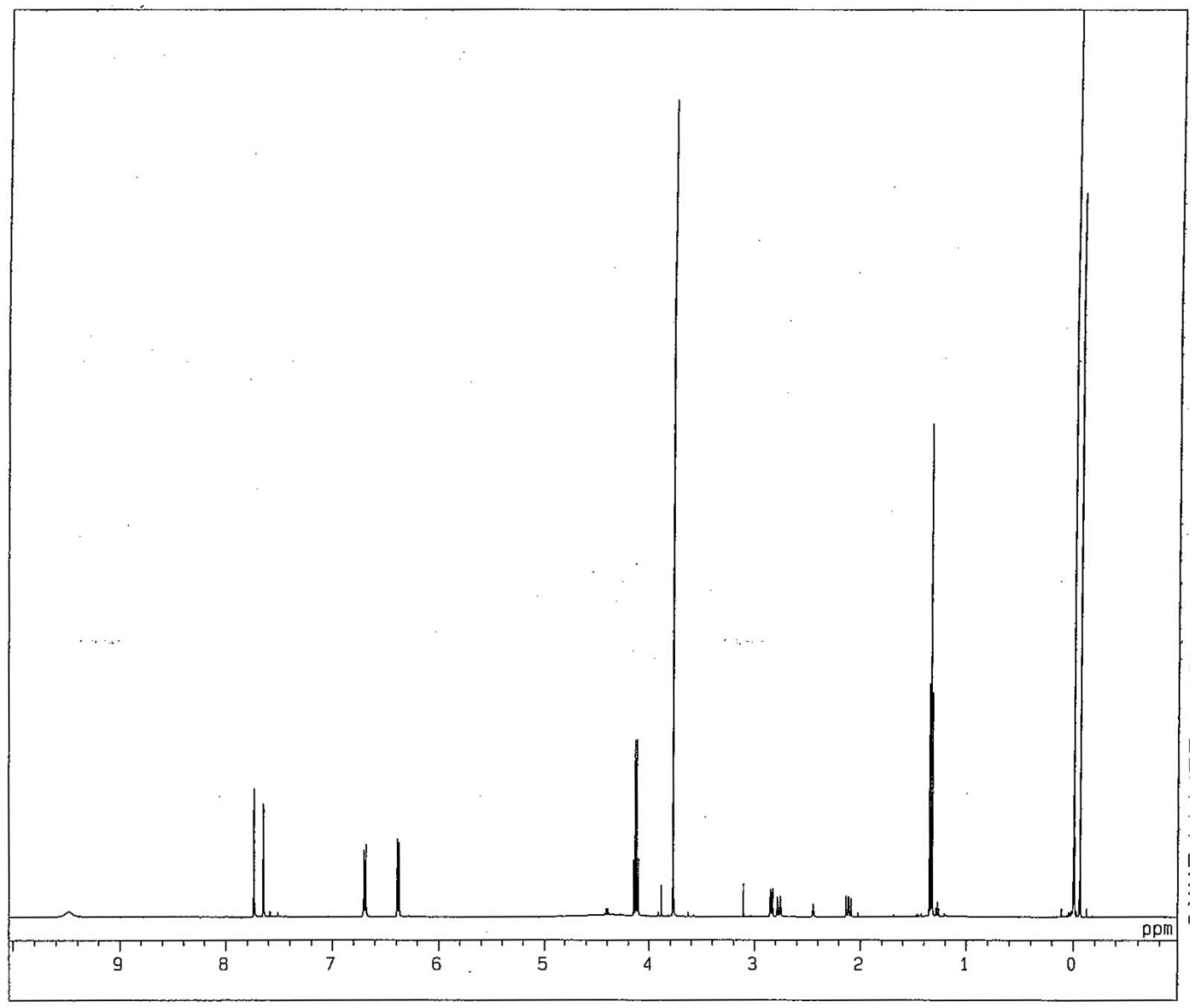


1-Ethyl-3-methylimidazolium 2-aminoisovaleric acid salt ([emim][Val]). From 1.0g (5.2mmol) of [emim] [Br], and 0.7g (6.1 mmol) of L-valine, $0.9 \mathrm{~g}$ of [emim][Val] was obtained (Yield : 79\%). ${ }^{1} \mathrm{H}-\mathrm{NMR}$ (DMSO, $\delta / \mathrm{ppm}$ relative to TMS) : ${ }^{1} \mathrm{H}-\mathrm{NMR}(\mathrm{DMSO}, \delta / \mathrm{ppm}$ relative to TMS): $0.63(\mathrm{~d}, 3 \mathrm{H}, \mathrm{J}=3.5 \mathrm{~Hz}$ ),

$0.76(\mathrm{~d}, 3 \mathrm{H}, \mathrm{J}=3.7 \mathrm{~Hz}), 1.36(\mathrm{t}, 3 \mathrm{H}, \mathrm{J}=7.5 \mathrm{~Hz}), 1.85(\mathrm{~m}, 1 \mathrm{H}, \mathrm{J}=22.5 \mathrm{~Hz}), 3.83(\mathrm{~s}, 3 \mathrm{H}), 4.17(\mathrm{q}, 2 \mathrm{H}, \mathrm{J}=11 \mathrm{~Hz})$, 7.72(s, 1H), 7.81(s, 1H), 9.79(s, 1H).

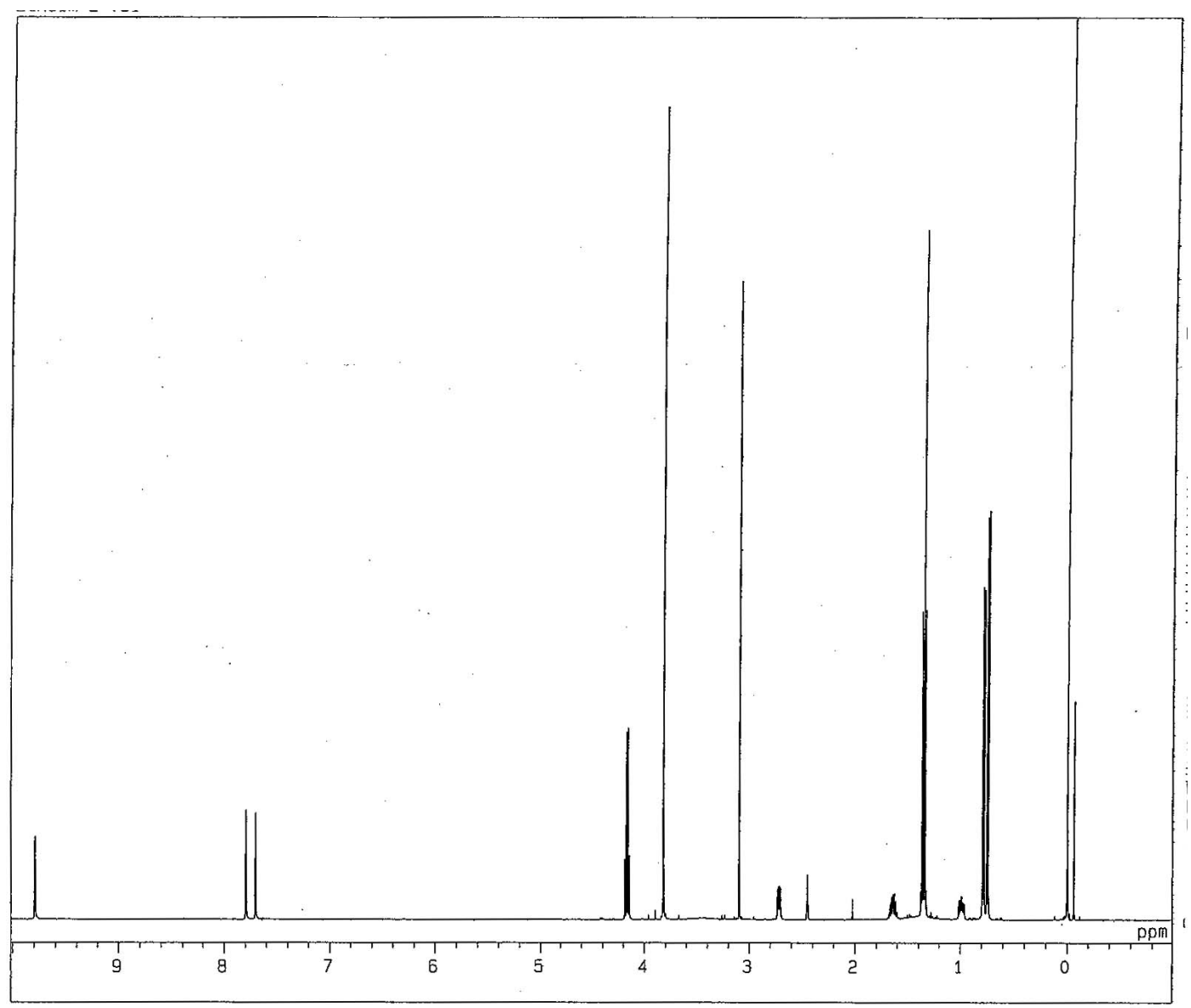

